selected visually and isolated by micromanipulation. The diploid status of selfed genotypes was confirmed by observing that they did not mate with tester strains of either mating type. For outcrossing, each asexual genotype was mated with a genotype from a parallel sexual population (for example, genotype 1 from replicate 1 of the sexual glucose population was mated with genotype 1 from replicate 1 of either the mating-type a or mating-type $\alpha$ asexual glucose population, depending on the mating type of the sexual genotype). Mating cultures were spread on glucose plates, and mated pairs of cells were selected by micromanipulation. The fitnesses $(w)$ of diploids produced by selfing and outcrossing were determined by competitions against a diploid produced by crossing yVB110 with a derivative of yVB114 carrying a URA3 genetic marker, and inbreeding depressions were calculated as $w_{\text {outcrossed }}-w_{\text {inbred }} / w_{\text {outcrossed }}$.

Received 23 April; accepted 9 May 1997

1. Bell, G. The Masterpiece of Nature (University of California, San Francisco, 1982).

2. Maynard Smith, J. The Evolution of Sex (Cambridge Univ. Press, 1978).

3. Kondrashov, A. S. Deleterious mutations and the evolution of sexual reproduction. Nature 336, 435 440 (1988).

4. Charlesworth, D., Morgan, T. M. \& Charlesworth, B. Mutation accumulation in finite outbreeding and inbreeding populations. Genet. Res. 61, 39-56 (1993).

5. Peck, J. R. A ruby in the rubbish: Beneficial mutations, deleterious mutations and the evolution of sex. Genetics 137, 597-606 (1994).

6. Malmberg, R. L. The evolution of epistasis and the advantage of recombination in populations of bacteriophage T4. Genetics 86, 607-621 (1977).

7. McPhee, C. P. \& Robertson, A. The effect of suppressing crossing-over on the response to selection in Drosophila melanogaster. Genet. Res. 16, 1-16 (1970).

8. Golemis, E. A., Gyuris, J. \& Brent, R. in Current Protocols in Molecular Biology (eds Ausubel, F. M. et al.) 13.1-13.14.17 (Wiley, Boston, 1994).

9. Birdsell, J. \& Wills, C. Significant competition advantage conferred by meiosis and syngamy in the yeast Saccharomyces cerevisiae. Proc. Natl Acad. Sci. USA 93, 908-912 (1996).

10. Kunz, B. A. \& Haynes, R. H. in The Molecular Biology of the Yeast Saccharomyces Vol. 1 (eds Strathern, J., Jones, E. W. \& Broach, J.) 371-414 (Cold Spring Harbor, Plainview, 1981).

11. Mortimer, R. K. \& Schild, D. in The Molecular Biology of the Yeast Saccharomyces Vol. 1 (eds Strathern, J., Jones, E. W. \& Broach, J.) 341-370 (Cold Spring Harbor, Plainview, 1981).

12. Menees, T. M. \& Sandmeyer, S. B. Transposition of the yeast retroviruslike element Ty3 is dependent on the cell cycle. Mol. Cell. Biol. 14, 8229-8240 (1994).

13. Muller, H. L. The relation of recombination to mutational advance. Mutat. Res. 1, $2-9$ (1964),

14. Crow, J. F. in The Evolution of Sex: an Examination of Current Ideas (eds Michod, R. E. \& Levin, B. R.) 56-73 (Sinauer, Sunderland, MA, 1988).

15. De Visser, J. A. G. M., Hoekstra, R. F. \& Van Den Ende, H. The effect of sex and deleterious mutations on fitness in Chlamydomonas. Proc. R. Soc. Lond. B 263, 193-200 (1996).

16. Bilanchone, V. W. et al. Positive and negative regulatory elements control expression of the yeast retrotransposon Ty3. Genetics 134, 685-670 (1993)

17. Herskowitz, I. \& Jensen, R. E. Putting the $H O$ gene to work: practical uses for mating-type switching. Methods Enzymol. 194, 132-146 (1990).

Acknowledgements. We thank S. Sandmeyer for yeast strains and plasmids; H. Bussey for plasmids and the use of his micromanipulator; H. Reiswig for the use of digitizing equipment; D. Schoen for comment on the manuscript; and A. Kondrashov for helpful criticism. This research was supported by NSERC an FACR grants to G.B., NSERC and FCAR scholarships to C.Z., and grants to R. Lenski.

\section{The heritability of IQ}

\section{B. Devlin ${ }^{\star}$, Michael Daniels $\uparrow$ \& Kathryn Roeder $\dagger$}

* Department of Psychiatry, University of Pittsburgh School of Medicine, Pittsburgh, Pennsylvania 15213, USA

$\dagger$ Department of Statistics, Carnegie Mellon University, Pittsburgh, Pennsylvania 15213, USA

IQ heritability, the portion of a population's IQ variability attributable to the effects of genes ${ }^{1}$, has been investigated for nearly a century, yet it remains controversial. Covariance between relatives may be due not only to genes, but also to shared environments, and most previous models have assumed different degrees of similarity induced by environments specific to twins, to non-twin siblings (henceforth siblings), and to parents and offspring. We now evaluate an alternative model that replaces these three environments by two maternal womb environments, one for twins and another for siblings, along with a common home environment. Meta-analysis of $\mathbf{2 1 2}$ previous studies shows that our 'maternal-effects' model fits the data better than the 'familyenvironments' model. Maternal effects, often assumed to be negligible, account for $20 \%$ of covariance between twins and $5 \%$ between siblings, and the effects of genes are correspondingly reduced, with two measures of heritability being less than $50 \%$. The shared maternal environment may explain the striking correlation between the IQs of twins, especially those of adult twins that were reared apart. IQ heritability increases during early childhood, but whether it stabilizes thereafter remains unclear. A recent study of octogenarians ${ }^{2}$, for instance, suggests that IQ heritability either remains constant through adolescence and adulthood $^{3}$, or continues to increase with age $^{2}$. Although the latter hypothesis has recently been endorsed ${ }^{4}$, it gathers only modest statistical support in our analysis when compared to the maternal-effects hypothesis. Our analysis suggests that it will be important to understand the basis for these maternal effects if ways in which IQ might be increased are to be identified.

Despite its conceptual simplicity, IQ heritability has engendered vitriolic debates throughout this century, debates that are now recurring ${ }^{5}$ following the publication of The Bell Curve by $\mathrm{R}$. Herrnstein and C. Murray ${ }^{6}$. Part of this controversy arises because IQ heritability is not well characterized. Paradoxically, direct versus indirect analytic methods, both of which attempt to estimate genetic effects on IQ unencumbered by environmental effects, yield markedly different estimates ${ }^{7}$. Direct studies presumably eliminate environmental covariance by evaluating relatives raised apart; indirect studies presumably eliminate environmental covariance by contrasting results from different study designs that have complementary environmental covariance terms. Contrary to their identical objectives, the former typically produces notably larger IQ heritability estimates. We postulate that this paradox is attributable in large part to the presence of unacknowledged maternal effects on IQ.

In traditional quantitative genetic studies, the environment is divided into maternal and external constituents ${ }^{1}$. We expand this dichotomy to take account of the realities of IQ adoption studies, lumping maternal environment and any shared external environment of adopted children into a single 'pre-separation environment'. Because the duration of shared external environment is usually small, pre-separation and maternal effects are roughly equivalent. Both are distinct from maternal inheritance, which is sometimes called a maternal effect ${ }^{8}$.

Our IQ analyses focus on the magnitude of the additive and nonadditive genetic components ${ }^{1}$, estimated to explain $60-85 \%$ of the variation in IQ from adult monozygotic twin studies, and the magnitude of maternal effects, usually assumed to be negligible. To estimate these effects, we analysed 212 IQ studies (or more precisely, correlations) based on 50,470 distinct pairings. The analysis included 204 correlations from studies of zero and firstdegree relatives or their adoptive counterparts ${ }^{9}$. We supplemented this set with some new twin studies published after 1981: a study of monozygotic twins reared apart ${ }^{10}$, the Swedish adoption/twin study of aging of monozygotic twins reared together and apart and dizygotic twins reared together ${ }^{11}$, and two studies of monozygotic and dizygotic adult twins reared together ${ }^{12}$.

Each IQ correlation and related sample size is classified by kind of study (Fig. 1). We evaluate these data using a standard quantitative genetic model for the components of variance (Table 1) and Bayesian meta-analysis ${ }^{13}$, a standard technique for combining information across studies. Our model is built on two levels of distributional assumptions: we assume a likelihood model for the observed correlations among relatives in each type of study; and we specify a prior distribution for the parameters of the model. We assume any standardized component of variance (positive correlation) is a priori equally likely to lie between zero and one. These prior distributions make the Bayesian parameter estimates similar to maximum-likelihood estimates.

All correlations were Fisher-transformed and, after transformation, observed correlations from the same study design follow a normal distribution if they differ only by measurement error. However, several studies are outliers under a normal likelihood, 
suggesting that the studies are more heterogeneous with respect to variability than is predicted by measurement error alone. Heterogeneity is expected because some study methods differ markedly'. We account for the heterogeneity by assuming that the transformed values follow a $t$-distribution with two degrees of freedom.

We fit four models to the data. The richest of these models, model IV, allows for additive and non-additive genetic effects, twin and singleton maternal effects, and external environmental effects for twins, siblings and parent-offspring. The remaining models differ from model IV in that Models I and II constrain the maternal effects to be zero, and models I and III constrain the external environmental effects to be equal.

The models are evaluated informally by comparing the average observed correlations for each of the nine study designs to the models' predicted values (Table 2). Model I predicts the correlation for the dizygotic twins raised together very poorly. Except for monozygotic twins raised apart, the predicted correlations for each study type are similar for models II-IV. The observed correlations for monozygotic twins raised apart (Fig. 1) ranges from 0.62 to 0.78 , with a mean of 0.74 . The most extreme predicted correlation is that obtained from model II (0.50), and the closest value is from model IV (0.74), but model III also predicts the correlation well, with a value of 0.68 . Maternal effects seem to be essential for a good fit to the data.

Models III and IV estimate a large maternal effect for twins and a smaller effect for siblings (Table 3). Model III attributes 20\% (95\% Bayesian credible interval: $15-24 \%)$ to the former and 5\% (1-8\%) to the latter. The difference between these estimates is intuitively appealing. Twins share the womb concurrently, whereas siblings share the womb serially. Hence, although a mother may have similar physiological status and personal habits from one pregnancy to another, the temporal separation between progeny apparently diminishes the correlation of sibling IQ.

\begin{tabular}{|c|c|c|c|}
\hline Relationship & Raised & Type (j) & Expected covariance \\
\hline $\begin{array}{l}\text { Monozygotic twins } \\
\text { Monozygotic twins } \\
\text { Dizygotic twins } \\
\text { Siblings } \\
\text { Siblings } \\
\text { Midparent/child } \\
\text { Single-parent/child } \\
\text { Single-parent/child } \\
\text { Adopting parent/child }\end{array}$ & $\begin{array}{l}\text { Together } \\
\text { Apart } \\
\text { Together } \\
\text { Together } \\
\text { Apart } \\
\text { Together } \\
\text { Together } \\
\text { Apart } \\
\text { Together }\end{array}$ & $\begin{array}{l}1 \\
2 \\
3 \\
4 \\
5 \\
6 \\
7 \\
8 \\
9\end{array}$ & $\begin{array}{c}\sigma_{A}^{2}+\sigma_{D}^{2}+\sigma_{M_{T}}^{2}+\sigma_{E S_{T}}^{2} \\
\sigma_{A}^{2}+\sigma_{D}^{2}+\sigma_{M_{T}}^{2} \\
\frac{1}{2}\left(1+r \sigma_{A}^{\dagger}\right) \sigma_{A}^{2}+\frac{1}{4} \sigma_{D}^{2}+\sigma_{M T}^{2}+\sigma_{E S_{T}}^{2} \\
\frac{1}{2}\left(1+r \sigma_{A}^{+}\right) \sigma_{A}^{2}+\frac{1}{4} \sigma_{D}^{2}+\sigma_{M_{S}}^{2}+\sigma_{E S_{S}}^{2} \\
\frac{1}{2}\left(1+r \sigma_{A}^{\dagger}\right) \sigma_{A}^{2}+\frac{1}{4} \sigma_{D}^{2}+\sigma_{M_{S}}^{2} \\
\sigma_{A}^{2}+\sigma_{E S_{P}}^{2} \\
\frac{1}{2}(1+r) \sigma_{A}^{2}+\sigma_{E S_{P}}^{2} \\
\frac{1}{2}(1+r) \sigma_{A}^{2} \\
\sigma_{E S_{P}}^{2}\end{array}$ \\
\hline
\end{tabular}

Midparent refers to the average of the two parents' IQ. Covariances are denoted by $\sigma^{2}$ with subscripts for genetic and environmental effects: $A$, additive genetic; $D$, nonadditive genetic; $G$, total genetic; $M_{T}$, maternal (twins); $M_{S}$, maternal (siblings); $E S_{T}, E S_{S}$ and $E S_{P}$ are environments for twins, siblings and parent/child, respectively; $T$, total. Standardized covariances, $\sigma_{k}^{2} / \sigma_{T}^{2}$, are denoted by $\sigma_{k}^{\dagger}$. Assortative mating is factored into the model by the correlation of IQs between mates, $r$. Following ref. 9, we take $r$ to be 0.33 (s.d. $=0.03$ ). Reanalysing the data with $r=0.33 \pm 2 \mathrm{~s} . \mathrm{d}$ had no notable effect on our findings.

Table 2 Posterior means for IQ correlations by study type

Model

\begin{tabular}{|c|c|c|c|c|c|c|c|}
\hline Relationship & Raised & Type & 0 & I & II & III & IV \\
\hline Monozygotic twins & Together & 1 & 0.85 & 0.85 & 0.85 & 0.85 & 0.85 \\
\hline Monozygotic twins & Apart & 2 & 0.74 & 0.68 & 0.50 & 0.68 & 0.74 \\
\hline Dizygotic twins & Together & 3 & 0.59 & 0.46 & 0.59 & 0.59 & 0.60 \\
\hline Siblings & Together & 4 & 0.46 & 0.46 & 0.44 & 0.44 & 0.44 \\
\hline Siblin & Apart & 5 & 0.24 & 0.28 & 0.23 & 0.27 & 0.28 \\
\hline Midparent/child & Together & 6 & 0.50 & 0.51 & 0.52 & 0.51 & 0.50 \\
\hline Single-parent/child & Together & 7 & 0.41 & 0.43 & 0.40 & 0.39 & 0.40 \\
\hline Single-parent/child & Apart & 8 & 0.24 & 0.25 & 0.23 & 0.22 & 0.21 \\
\hline Adopting parent/child & Together & 9 & 0.20 & 0.18 & 0.17 & 0.17 & 0.18 \\
\hline
\end{tabular}

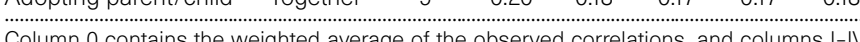
Column 0 contains the weighted average of the observed correlations, and columns I-IV contain the predicted values of these correlations from models I-IV. The predicted correlations are obtained through a Bayesian simulation procedure that evaluates integrals numerically ${ }^{14}$
Remarkably, the covariance attributed to environmental components is also roughly equal for models II-IV (Table 3 ). The notable difference among the models is how the covariance resulting from shared environments is partitioned.

The models are compared formally using Bayes factors ${ }^{15,16}$ to obtain their posterior probabilities. A priori each model is assumed equally likely. The best model is model III, with a posterior probability of 0.95 , followed by model IV, with probability 0.05 , and models I and II, with probabilities near zero. Hence model $\mathrm{II}^{17}$, which is commonly used to describe the quantitative genetics of IQ, is rejected when it is compared to model III, which involves a common home environment and a maternal environment that varies depending on whether the pair are twins or not. Model III is favoured over model II by a posterior factor of almost 10,000 to 1 , primarily on account of model II's fit to the observed data from monozygotic twins reared apart: its predicted value is about six standard deviations from the mean. Model IV, which combines features of models II and III, seems to be overparameterized and its environmental components make little sense (Table 3).

Model III's parameter estimates (Table 3) define estimates of IQ heritability. However, the concept of heritability has narrow-sense and broad-sense interpretations. Narrow-sense heritability accounts for additive genetic effects only; broad-sense heritability encompasses both additive and non-additive genetic effects. This distinction is often lost in the 'IQ debates', even though it is critical to the social implications of IQ heritability ${ }^{18}$. Based on model III, broad- and narrow-sense heritability estimates are $48 \%$ and $34 \%$, respectively; both agree closely with estimates from studies using standard quantitative genetic models ${ }^{19,20}$ and indirect estimation methods ${ }^{7,21}$.

An IQ enigma has been developed ${ }^{7}$ by contrasting the typically larger direct heritability estimates with complementary, smaller indirect estimates. For example, an indirect estimate of broad-

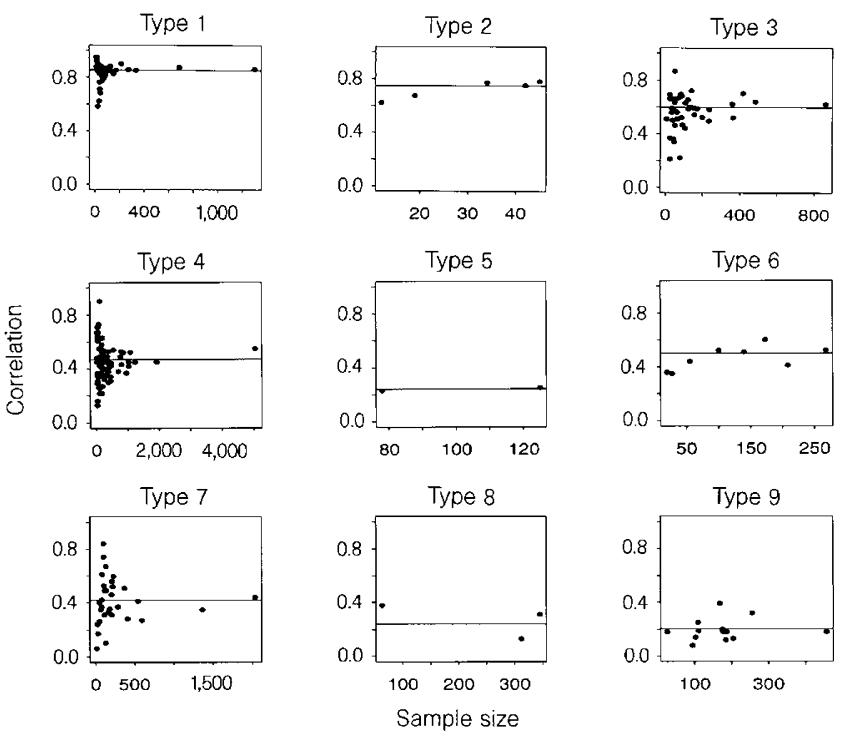

Figure 1 IQ correlations for certain study designs (see Table 1 for types) plotted versus sample size. The horizontal line indicates the mean correlation. Note the scatter about each mean is consistent with decreasing variance with increasing sample size, suggesting that much of the variance in the IQ correlations is due to sampling variance. Four type 4 studies, with missing sample size, are assigned the median sample size of 201. We analyse all study types ${ }^{6}$ with zero or firstdegree relatives and their adoptive counterparts with the exception of adopted/ adopted siblings. Adopted/adopted studies are excluded because the observed correlations are extremely variable relative to the other sets of correlations, and are inconsistent with the remaining data. If these data are included in our analysis, there is no appreciable difference in variance components estimates. 


\begin{tabular}{|c|c|c|c|c|c|}
\hline \multirow[b]{2}{*}{ Effect } & \multirow[b]{2}{*}{ Notation } & & & & \\
\hline & & I & II & III & IV \\
\hline Additive genetic & $\sigma_{A}^{\dagger}$ & $0.33(0.27,0.39)$ & $0.35(0.28,0.41)$ & $0.34(0.27,0.40)$ & $0.32(0.26,0.39)$ \\
\hline Non-additive genetic & $\sigma_{D}^{\dagger}$ & $0.34(0.31,0.38)$ & $0.15(0.10,0.21)$ & $0.15(0.09,0.20)$ & $0.15(0.10,0.21)$ \\
\hline total genetic & $\sigma_{G}^{+}$ & $0.68(0.64,0.71)$ & $0.50(0.45,0.56)$ & $0.48(0.43,0.54)$ & $0.47(0.42,0.52)$ \\
\hline Maternal (twins) & $\sigma_{M_{T}}^{\dagger}$ & & & $0.20(0.15,0.24)$ & $0.27(0.18,0.35)$ \\
\hline Maternal (siblings) & $\sigma_{M_{S}}^{M_{T}}$ & & & $0.05(0.01,0.08)$ & $0.06(0.00,0.17)$ \\
\hline Environment (common) & $\begin{array}{l}M_{S} \\
\sigma_{E S}\end{array}$ & $0.17(0.13,0.21)$ & & $0.17(0.13,0.21)$ & \\
\hline Environment (twins) & $\sigma_{E S_{T}}^{\dagger}$ & & $0.35(0.30,0.41)$ & & $0.11(0.05,0.19)$ \\
\hline Environment (siblings) & $\sigma_{E S_{S}}^{\dagger}$ & & $0.21(0.17,0.25)$ & & $0.17(0.06,0.24)$ \\
\hline Environment (parent/child) & $\sigma_{E S_{p}}^{\dagger}$ & & $0.17(0.13,0.21)$ & & $0.18(0.14,0.23)$ \\
\hline
\end{tabular}

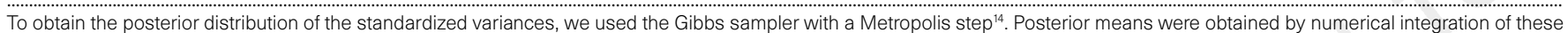
distributions, as were the credible intervals for the parameter estimates.

sense heritability is obtained by multiplying twice the difference between monozygotic and dizygotic twin correlations (Table 2). For these data (Table 3 ), the broad-sense heritability estimate of 0.52 is slightly greater than that obtained from model III, which is expected because the contrast overestimates heritability by about $0.5 \sigma_{D}^{\dagger}$ (see Table 1). The correlation for monozygotic twins reared apart yields a much larger direct estimate of 0.74 . The difference between estimates can be reduced by subtracting the maternal effect from the direct estimate, yielding a value of 0.54 .

Twice the observed correlation for siblings reared apart yields another direct, biased estimate of broad-sense heritability; the bias is $-1 / 2 \sigma_{D}^{\dagger}$, estimated from model III to be $-7.5 \%$. This direct estimate, 0.48 , is much lower than the direct estimate obtained from the monozygotic twins reared apart, but it is similar to the indirect estimate and model III's estimate. The similarity to model III's estimate can be attributed to the balance between the bias and the 5\% sibling maternal effect, which the direct estimates ignores. Thus maternal effects may resolve the IQ enigma by rectifying disparate direct and indirect heritability estimates.

It is not difficult to gather evidence for the importance of maternal environment. There is substantial brain growth in utero, and the brain has $70 \%$ of its final mass within a year of birth. Additionally, various studies indicate that IQ can be affected by prenatal environment: IQ is positively correlated with birth weight even after controlling for gestational age $\mathrm{e}^{22,23}$; twins, who usually weigh less than singletons, average 4-7 points lower on IQ tests ${ }^{24}$; some dietary supplements can raise IQ $s^{25,26}$; alcohol, drug and cigarette consumption may lower IQ $s^{27,29}$; and lead exchange from mother to fetus may reduce $\mathrm{IQ}^{30}$.

In contrast, the large direct estimates derived from studies of adult twins raised apart have been explained by the conjecture that, as twins get older, IQ becomes more heritable. Evidence has been provided to support this 'age hypothesis' ${ }^{4,12}$, although the results are inconclusive and relationship unclear.

To compare the age- and maternal-effects hypotheses, we related the IQ correlations to subject age whenever possible. We classified the studies by age into three categories, namely youths ( $<14$ years), adolescents (14-18 years) and adults ( $>18$ years), based on median or mean subject age for each study; we assigned $71 \%$ of the twin and sibling studies. Because they are cross-generational, assigning parent-offspring studies was problematic: in one treatment we included all such studies as adolescents, and in another we excluded all such studies except for parent/child apart. We then extended model II. This age-effect model allowed heritability to increase with age by two adjustments: a multiplicative increase in the genetic components of variance, namely $b^{-1 / 2}$ for youths, $b^{0}$ for adolescents, and $b^{2}$ for adults; and a multiplicative decrease in the shared environment components of variance, namely $c^{1 / 2}$ for youths, $c^{0}$ for adolescents, and $c^{-2}$ for adults. For $b$ and $c>1$, these multiplicative functions have forms consistent with those hypothesized in ref. 12. Our prior distribution for $b$ and $c$ favoured the age hypothesis slightly by putting uniform mass between $(1 / \sqrt{ } 2, \sqrt{2})$.
The parameter estimates for $b(1.13,[1.05-1.19])$ and $c$ $(1.21,[1.03-1.38])$, based on the analysis of all types of study, are consistent with the age hypothesis. Because Bayes factors (BF) impose a penalty for added complexity we also considered a oneparameter age-effect model with $b=c$. This model is favoured over the two-parameter model $(B F=2.5)$, and is also favoured to a small degree over model II $(B F=11, b=1.10$, [1.04-1.16]). Similar results are obtained when parent-offspring studies are excluded. However, no version of model II is competitive with model III by Bayes factor. Moreover, age-effects models fail to fit the data better than a simpler model that invokes maternal effects.

Our statistical analyses cannot, of course, be considered definitive. Age may affect IQ heritability, even though the age model does not gain much support from our analyses. Moreover, our analyses do not preclude other, unmodelled factors, such as cultural inheritance and interaction between genes and the environment, from having important effects on IQ. Despite these caveats though, several important conclusions emerge from our results.

Adoption designs are a popular means of estimating IQ heritability. Associated analyses, however, usually assume negligible maternal effects. By contrast, our results show that $20 \%$ of twin and $5 \%$ of sibling covariance may be attributable to maternal effects. These results have two implications: a new model may be required regarding the influence of genes and environment on cognitive function; and interventions aimed at improving the prenatal environment could lead to a significant increase in the population's IQ. Moreover, some of Herrnstein and Murray's conclusions ${ }^{6}$ regarding human evolution such as the development of cognitive castes and IQ dysgenics, arise from their belief that IQ heritability is at least $60 \%$, and is probably closer to the $80 \%$ values obtained from adoption studies. Our results suggest far smaller heritabilities: broad-sense heritability, which measures the total effect of genes on IQ, is perhaps $48 \%$; narrow-sense heritability, the relevant quantity for evolutionary arguments because it measures the additive effects of genes, is about 34\%. Herrnstein and Murray's evolutionary conclusions are tenuous in light of these heritabilities ${ }^{18}$.

Received 12 February; accepted 17 June 1997

1. Falconer, D. S. Introduction to Quantitative Genetics (Longman, New York, 1981)

2. McClearn, G. E. et al. Substantial genetic influence on cognitive abilities in twins 80 or more years old. Science 276, 1560-1563 (1997).

Gottesman, I. I. Twins: en route to QTLs for cognition. Science 276, 2522-1523 (1997).

4. Task Force for Scientific Affairs. Intelligence: Knowns and Unknowns (American Psychological Association, Washington DC, 1995)

5. Devlin, B., Fienberg, S., Resnick, D. \& Roeder, K. (eds) Intelligence, Genes and Success: Scientists Respond to The Bell Curve (Springer, New York, 1997).

6. Herrnstein, R. J. \& Murray, C. The Bell Curve: Inteligence and Class Structure in American Life (Free Press, New York, 1994).

7. Plomin, R. \& Loehlin, J. C. Direct and indirect IQ heritability studies: a puzzle. Behav. Genet. 19, 331342 (1989).

8. Rose, R. J. et al. Data from kinships of monozygotic twins indicate maternal effects on intelligence. Nature 283, 375-377 (1980).

9. Bouchard, T. J. Jr \& McGue, M. Familial studies of intelligence: a review. Science 250, 223-228 (1990). 10. Bouchard, T. J. Jr, Lykken, D. T., McGue, M., Segal, N. L. \& Tellegen, A. Sources of human psychological differences: the Minnesota study of twins reared apart. Science 250, 223-228 (1990).

11. Pedersen, N. L., Plomin, R., Nesselroade, J. R. \& McClearn, G. E. A quantitative genetic analysis of cognitive abilities during the second half of the life span. Psychol. Sci. 3, 346-353 (1992). 
12. McGue, M., Bouchard, T. J. Jr, Jacono, W. G. \& Lykken, D. T in Nature, Nurture and Psychology (eds Plomin, R. \& McClearn, G. E.) 59-76 (American Psychological Association, Washington DC, 1993) 13. National Research Council. Combining Information. Statistical Issues and Opportunities for Research (National Academy Press, Washington DC, 1992).

14. Smith, A. F. M. \& Roberts, G. O. Bayesian computation via the Gibbs sampler and related Markov chain Monte Carlo methods. J. R. Stat. Soc. B 55, 3-24 (1993).

15. Kass, R. E. \& Raftery, A. E. Bayes Factors. J. Am. Stat. Assoc. 90, 773-795 (1995).

16. Verdinelli, I. \& Wasserman, L. Computing Bayes factors by using a generalization of the Savage-Dickey density ratio. J. Am. Stat. Assoc. 90, 614-618 (1995).

17. Plomin, R., DeFries, J. C. \& McClearn, G. E. Behavioral Genetics: A Primer (Freeman, New York, 1990).

18. Daniels, M., Devlin, B. \& Roeder, K. in Intelligence, Genes and Success: Scientists respond to The Bell Curve (eds Devlin, B., Fienberg, S., Resnick, D. \& Roeder, K.) (Springer, New York, 1997).

19. Chipuer, H. M., Rovine, M. J. \& Plomin, R. LISREL modeling: genetic and environmental influences on IQ revisited. Intelligence 14, 11-29 (1990).

20. Rao, D. C., Morton, N. E., Lalouel, J. M. \& Lew, R. Path analysis under generalized assortative mating. II. American IQ. Genet. Res. 39, 187-198 (1982).

21. McCartney, K., Harris, M. J. \& Bernieri, F. Growing up and growing apart: a developmental metaanalysis of twin studies. Psychol. Bull. 107, 226-237 (1990).

22. Lynn, R \& Hattori, K. The heritability of intelligence in Japan. Behav. Genet 20, 545-546 (1990).

23. Churchill, J. A. The relationship between intelligence and birth weight in twins. Neurology 15, 341347 (1965).

24. T. Husén, T. Intra-pair similarities in the school achievement of twins. Scand. J. Psychol. 4, 108-144 (1963).

25. Harrell, R. F., Woodyard, E. \& Gates, A. I. The Effects of Mothers' Diets on the Intelligence of Offspring (Teacher's College, New York, 1955)

26. Rush, D., Stein, Z., Susser, M. \& Brody, N. in Diet in Pregnancy: A Randomized Controlled Trial of Nutritional Supplements (eds Rush, D., Stein, Z. \& Susser, M.) 88-131 (Liss, New York, 1980).

27. Reinisch, J., Sanders, S. A., Mortensen, E. L. \& Rubin, D. B. In utero exposure to phenobarbital and intelligence deficits in adult men. J. Am. Med. Assoc. 274, 1518-1525.

28. Streissguth, A. P., Barr, H. M., Sampson, P. D., Darby, B. L. \& Martin, D. C. IQ at age 4 in relation to maternal alcohol and smoking during pregnancy. Dev. Psychol. 25, 3-11 (1989).

29. Olds, D. L., Henderson, C. R. Jr \& Tatelbaum, R. Intellectual impairment in children of women who smoke cigarettes during pregnancy. Pediatrics 93, 221-227 (1994)

30. Baghurst, P. A. et al. Environmental exposure to lead and children's intelligence at the age of seven years. N. Engl. J. Med. 327, 1279-1284 (1992).

Acknowledgements. We thank T. J. Bouchard Jr for compilations of IQ data, and J. C. Loehlin, M. McGue, S. Fienberg and R. Kass for comments on a previous draft of the manuscript. This work was supported by grants from the National Science Foundation and the National Institutes of Health.

Correspondence and requests for materials should be addressed to B.D. (e-mail: devlinbj@msx.upmc. edu).

\section{Second-order fear} conditioning prevented by blocking NMDA

\section{receptors in amygdala}

\section{Jonathan C. Gewirtz \& Michael Davis}

Departments of Psychology \& Psychiatry, Yale University, Ribicoff Research Facilities of the Connecticut Mental Health Center, 34 Park Street, New Haven, Connecticut 06508, USA

Antagonists of NMDA ( $N$-methyl-D-aspartate)-type glutamate receptors disrupt several forms of learning ${ }^{1-8}$. Although this might indicate that NMDA-receptor-mediated processes are critical for synaptic plasticity, there may be other mechanisms by which NMDA-receptor antagonism could interfere with learning ${ }^{1,9-12}$. For instance, fear conditioning would be blocked by microinfusion of the NMDA-receptor antagonist AP5 (D,L-2amino-5-phosphonovalerate) into the basolateral amygdala ${ }^{6,13,14}$ if AP5 inhibited routine synaptic transmission, thereby reducing the ability of stimuli to activate amygdala neurons ${ }^{15,16}$. In secondorder fear conditioning ${ }^{17,18}$, the reinforcer is a fear-eliciting conditioned stimulus rather than an unconditioned stimulus. Expression of conditioned fear is amygdala-dependent ${ }^{19,20}$ and so provides a behavioural assessment of the ability of the reinforcer to activate amygdala neurons in the presence of AP5. We report here that intra-amygdala AP5 actually enhances expression of conditioned fear to the conditioned stimulus that provides the reinforcement signal for second-order conditioning. Nevertheless, acquisition of second-order fear conditioning is completely blocked. Our findings strongly support the view that NMDA receptors are critically involved in synaptic plasticity.

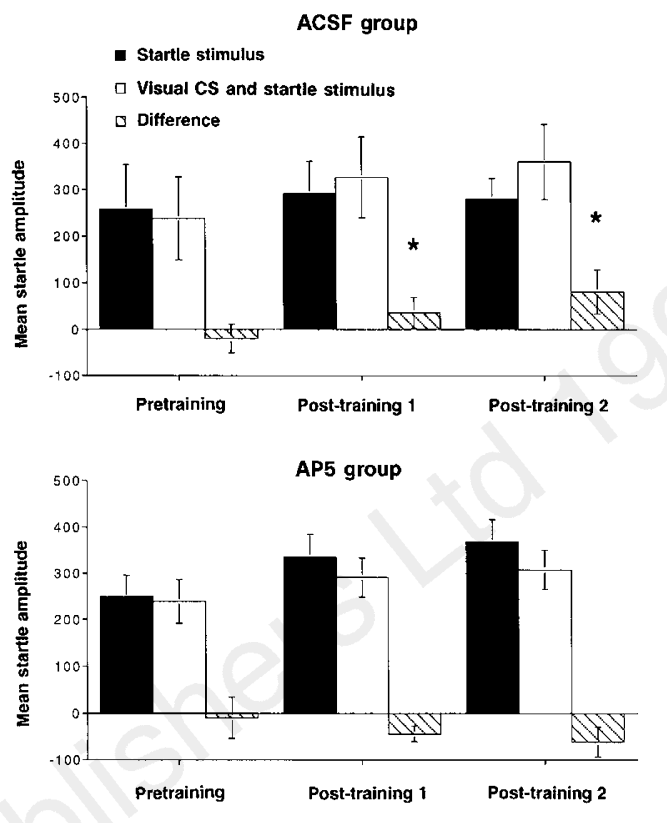

Figure 1 Fear-potentiated startle to the second-order CS in ACSF (top) and AP5 (bottom) groups tested before second-order conditioning training, and after two (post-training 1), and three (post-training 2) sessions of second-order conditioning training. Fear-potentiated startle (hatched bars, \pm s.e.m.) was calculated as the difference between startle amplitude in the presence (white bars, \pm s.e.m.) and absence (black bars, \pm s.e.m.) of the visual CS. No infusion was given immediately before tests. An analysis of variance (ANOVA) revealed a significant group- $x$ session interaction, $F(2,34)=3.8, P<0.05$. In the ACSF group, fearpotentiated startle increased significantly relative to pretraining baseline in posttraining tests 1 and 2 ; values of $t(8)$ were 2.4 and 2.6 respectively, $P<0.05$. In the AP5 group, the CS actually tended to inhibit startle relative to the pretraining baseline in post-training tests 1 and 2 , although this effect was not significant; $t(9)$ was 0.7 and 1.3 , respectively.

Pavlovian fear conditioning seems to involve the convergence of inputs representing the conditioned stimulus (CS) and the unconditioned stimulus (US) in the basolateral amygdala ${ }^{21}$. Microinfusion of NMDA receptor antagonists into the amygdala blocks the acquisition of fear conditioning, measured using the fearpotentiated startle response $e^{6,13}$. This is consistent with data from other behavioural models suggesting that NMDA receptor activation is critical for the synaptic plasticity underlying several forms of memory ${ }^{1-8}$. Alternatively, NMDA receptor blockade might interfere with the ability of the CS or the US to activate cells in the amygdala $^{15,16}$. It is unlikely that the ability of the CS to activate amygdala neurons is impaired, because local infusion of NMDA antagonists, in contrast to AMPA antagonists ${ }^{19}$, does not interfere with the expression of fear conditioning previously acquired ${ }^{6,13}$. However, the ability of the US (typically foot shock) to activate amygdala neurons is not behaviourally testable in this learning protocol.

In pavlovian second-order fear conditioning, a second-order CS acquires conditioned fear by being paired with a first-order CS that has been paired previously with foot shock. In this case, the ability of the reinforcer to activate the amygdala can be assessed by the degree to which the first-order CS elicits conditioned fear. Thus, secondorder conditioning can be used to evaluate whether an NMDAreceptor antagonist blocks acquisition of conditioned fear or interferes with synaptic transmission of the reinforcement signal in the amygdala.

To test this, male albino rats were implanted with bilateral cannulae aimed at the basolateral amygdala. In four sessions of 